\title{
Computing complexity measures for quantum states based on exponential families
}

\author{
Sönke Niekamp ${ }^{€}$, Tobias Galla ${ }^{£}$, Matthias Kleinmann ${ }^{€}$, and \\ Otfried Gühne $€$ \\ $€$ Naturwissenschaftlich-Technische Fakultät, Universität Siegen, \\ Walter-Flex-Straße 3, D-57068 Siegen, Germany \\ $£$ Theoretical Physics, School of Physics and Astronomy, The University of \\ Manchester, Manchester M13 9PL, United Kingdom
}

\begin{abstract}
Given a multiparticle quantum state, one may ask whether it can be represented as a thermal state of some Hamiltonian with $k$-particle interactions only. The distance from the exponential family defined by these thermal states can be considered as a measure of complexity of a given state. We investigate the resulting optimization problem and show how symmetries can be exploited to simplify the task of finding the nearest thermal state in a given exponential family. We also present an algorithm for the computation of the complexity measure and consider specific examples to demonstrate its applicability.

E-mail: soenke.niekamp@uni-siegen.de, tobias.galla@manchester.ac.uk, matthias.kleinmann@uni-siegen.de, otfried.guehne@uni-siegen.de
\end{abstract}

\section{Introduction}

Understanding interacting multiparticle systems is a central problem in many areas of physics, including condensed matter theory, quantum information processing, and complexity science. The difficulty of this problem arises from correlations between the particles: Typically, probability distributions of states of two or more particles do not factorize, hence the description of interacting systems requires significantly more parameters than the description of the same number of non-interacting particles. This makes the analysis of multiparticle systems challenging, but it also leads to novel and interesting phenomena such as quantum entanglement and classical complex behaviour.

There are many approaches with which to characterize the complexity and correlations of multiparticle systems. In quantum information theory, much research has focused on entanglement measures [1 3], but also on other forms of quantum correlations [4, 5]. Similarly, a number of different measures of complexity have been introduced for classical complex systems [6 11].

An approach which can be used to measure complexity in the classical as well as in the quantum domain makes use of interaction structures [12 15]. In this approach one asks: Given a physical system, can its stationary state or density operator be viewed as 
a thermal state of an interacting particle system with $k$-body interactions only? If this is not the case, then how far is the state of the system from the space of all thermal states with $k$-body interactions? This distance can then be used as a measure of complexity. To measure distances of this type it is useful to make use of the relative entropy, and of the underlying geometrical structure known in mathematics as information geometry [16]. As already mentioned, an important feature of this approach is the fact that it can be used for the quantum and the classical case: In classical multiparticle systems, each particle is assumed to be on one of a finite set of states at any one time, and the global physical system is described by a probability distribution over the products of the local states. In the spirit of Ref. [12] interactions are described by the Hamiltonian function on the state space, where a Hamiltonian is a $k$-particle Hamiltonian, if it is a sum of functions each of which acts on $k$-particles only. It is important to stress though that the Hamiltonian used in this approach is not necessarily a physical energy function, it is mostly a mathematical object with which to characterise the factorisation properties of the system's stationary state. Indeed, the approach is applicable to non-equilibrium systems as well, for which there may not be any energy function at all. In the quantum case, the physical system is described by a density matrix, and the Hamiltonian is an operator acting on the corresponding Hilbert space, but again it need not be that of an actual physical system.

A central problem for the practical application of this approach in the quantum case is the calculation of the respective distances. For classical systems with binary states efficient algorithms are known [15,17, 18]. For the quantum case, some analytical results for states with a high degree of symmetry have been derived [13]. Moreover, an algorithmic approach has been proposed in Ref. [19]. Nevertheless the practical applicability of these ideas is not clear at present, and more general results and an overarching mathematical theory are as yet missing.

In this paper, we present several results relating to the computation of the distance of a given quantum state from the set of thermal states generated by Hamiltonians with $k$-particle interactions. First, we show how symmetries of the state can be used to simplify calculations of this type. Second, we present a new algorithm for the efficient computation of such distances. We also discuss how tools from convex optimization can be used in order to compute complexity measures for quantum states.

Our paper is organized as follows: In Section 2, we introduce the relevant notation, in particular the formalism of quantum exponential families, and we explain the most relevant existing results. In Section 3 we show that for cases in which the quantum state being studied carries a certain symmetry the closest thermal state generated by $k$-particle Hamiltonians has the same symmetry. In Section 4 we present our algorithm and discuss its application to specific examples. In Section 5 we discuss how results from optimization theory can be used to study this problem. Section 6 finally summarises our findings and we present an outlook on future lines of research. 


\section{Exponential families of quantum states}

In this section we introduce the theory of exponential families of interaction spaces for the special case of quantum states. We emphasize that most of the results presented here have been derived for the classical case by various authors (see, e.g., Refs. [12,16, 20]) and for the quantum case mostly by D. L. Zhou [13,14]. Nevertheless, since these results are rather scattered in the literature, we believe that a more comprehensive presentation can be useful.

\subsection{Exponential and Bloch representation}

We consider systems consisting of $n$ two-level systems (qubits) throughout; the generalization to higher-dimensional systems is straightforward. For later convenience, we first describe two different ways of representing quantum states of such systems.

The first possible representation is the exponential representation. It uses the fact that any quantum state can be considered as a thermal state of some appropriately chosen Hamiltonian. More precisely, any $n$-qubit quantum state of full rank can be written as

$$
\varrho_{\exp }(\boldsymbol{\theta})=\exp \left(\sum_{\alpha_{1}, \ldots, \alpha_{n}} \theta_{\alpha_{1}, \ldots, \alpha_{n}} \sigma_{\alpha_{1}} \otimes \cdots \otimes \sigma_{\alpha_{n}}\right)
$$

where the indices $\alpha_{k}$ run from 0 to 3 and the $\sigma_{i}$ are the Pauli matrices with the convention $\sigma_{0}=\mathbb{1}, \sigma_{1}=\sigma_{x}, \sigma_{2}=\sigma_{y}$ and $\sigma_{3}=\sigma_{z}$. In the following, it will be convenient to use a multi-index notation,

$$
\varrho_{\exp }(\boldsymbol{\theta})=\exp \left(\sum_{\alpha} \theta_{\alpha} \sigma_{\alpha}\right)
$$

where $\sigma_{\alpha}=\sigma_{\alpha_{1}} \otimes \cdots \otimes \sigma_{\alpha_{n}}$, and where $\alpha=\left(\alpha_{1}, \ldots, \alpha_{n}\right)$. The coefficient $\theta_{0}$ of the identity $\sigma_{0}=\mathbb{1}^{\otimes n}$ in the above quantum states is not arbitrary, as it can be determined from the normalization condition $\operatorname{tr} \varrho_{\exp }(\boldsymbol{\theta})=1$. Explicitly one has $\theta_{0}=-\psi(\boldsymbol{\theta})$, where

$$
\psi(\boldsymbol{\theta})=\ln \left\{\operatorname{tr}\left[\exp \left(\sum_{\alpha \neq 0} \theta_{\alpha} \sigma_{\alpha}\right)\right]\right\}
$$

Any quantum state, $\varrho$, of full rank can be written in the form of Eq. (1), and one can view the exponent in the exponential representation as a Hamiltonian of which $\varrho$ is the thermal state. In this terminology, the function $\psi$ is up to a sign the free energy of statistical ensemble [21 23]. It is important to note, however, that the Hamiltonian does not necessarily correspond to that of an actual physical system.

An alternative description of the quantum state is given by the affine representation or Bloch representation. In this representation one writes the state as

$$
\varrho_{\text {aff }}(\boldsymbol{\eta})=\frac{1}{2^{n}} \sum_{\alpha} \eta_{\alpha} \sigma_{\alpha},
$$

where the coefficients are given by $\eta_{\alpha}=\operatorname{tr}\left(\varrho_{\text {aff }} \sigma_{\alpha}\right)$. Here, the normalization condition is simply given by $\eta_{0}=1$. Note that in the affine representation the positivity of the density matrix results in additional restrictions on the coefficients $\boldsymbol{\eta}$; these conditions, however, cannot normally be formulated straightforwardly [24, 25]. 
We will now briefly discuss the connections between the two representations of quantum states. In order to do so consider two states $\varrho$ and $\varrho^{\prime}$ of full rank in their different representations, $\varrho=\varrho_{\exp }(\boldsymbol{\theta})=\varrho_{\text {aff }}(\boldsymbol{\eta})$ and $\varrho^{\prime}=\varrho_{\exp }\left(\boldsymbol{\theta}^{\prime}\right)=\varrho_{\text {aff }}\left(\boldsymbol{\eta}^{\prime}\right)$. Using the standard definition $\ddagger$ of the relative entropy between two quantum states, $\varrho$ and $\chi$,

$$
D(\varrho \| \chi)=\operatorname{tr}\left[\varrho \log _{2}(\varrho)\right]-\operatorname{tr}\left[\varrho \log _{2}(\chi)\right]
$$

as well as the entropy $S(\varrho)=-\operatorname{tr}\left(\varrho \log _{2} \varrho\right)$ of a single quantum state, we then have

$$
\begin{aligned}
\ln (2) D\left(\varrho \| \varrho^{\prime}\right) & =-\ln (2) S\left[\varrho_{\text {aff }}(\boldsymbol{\eta})\right]-\operatorname{tr}\left\{\frac{1}{2^{n}}\left[\mathbb{1}+\sum_{\alpha \neq 0} \eta_{\alpha} \sigma_{\alpha}\right]\left[\sum_{\beta \neq 0} \theta_{\beta}^{\prime} \sigma_{\beta}-\psi\left(\boldsymbol{\theta}^{\prime}\right) \mathbb{1}\right]\right\} \\
& =\phi(\boldsymbol{\eta})+\psi\left(\boldsymbol{\theta}^{\prime}\right)-\sum_{\alpha \neq 0} \eta_{\alpha} \theta_{\alpha}^{\prime}
\end{aligned}
$$

where the function $\phi(\boldsymbol{\eta})=-\ln (2) S\left[\varrho_{\text {aff }}(\boldsymbol{\eta})\right]=-\ln (2) S(\varrho)$ is proportional to the entropy of $\varrho$. With the scalar product $\boldsymbol{\eta} \cdot \boldsymbol{\theta}^{\prime}=\sum_{\alpha \neq 0} \eta_{\alpha} \theta_{\alpha}^{\prime}$ this result takes the form

$$
\ln (2) D\left(\varrho \| \varrho^{\prime}\right)=\phi(\boldsymbol{\eta})+\psi\left(\boldsymbol{\theta}^{\prime}\right)-\boldsymbol{\eta} \cdot \boldsymbol{\theta}^{\prime}
$$

For the special case in which $\varrho=\varrho^{\prime}$ this reads

$$
\phi(\boldsymbol{\eta})+\psi(\boldsymbol{\theta})-\boldsymbol{\eta} \cdot \boldsymbol{\theta}=0,
$$

a result which will become important below. At this point it should be noted that the expression of Eq. (8) shows that $\psi(\boldsymbol{\theta})$ and $\phi(\boldsymbol{\eta})$ are related by a Legendre transformation. More specifically, from Eq. (8) it follows that $\eta_{\alpha}=\partial \psi(\boldsymbol{\theta}) / \partial \theta_{\alpha}$ and $\theta_{\alpha}=\partial \phi(\boldsymbol{\eta}) / \partial \eta_{\alpha}$ for all $\alpha \neq 0$.

Similar structures are, of course, well known in statistical mechanics: a thermodynamic ensemble in statistical mechanics is defined by the requirement that some observables $A_{i}$ (e.g. the Hamiltonian) have fixed expectation values (e.g. the internal energy $U$ ). Maximizing the entropy of the statistical distribution of the ensemble under these constraints, the thermal state of the ensemble comes out as $\varrho \sim \exp \left(-\sum_{i} \lambda_{i} A_{i}\right)$, where the coefficients $\lambda_{i}$ arise as Lagrange multipliers. It is then well-known that the Lagrange multipliers $\lambda_{i}$ are related to the expectation values $\left\langle A_{i}\right\rangle$ by a Legendre transformation (see page 40 in Ref. [21], or [22, 23]).

Let us finally explain a useful theorem for the relative entropies between three states. For the pairwise relative entropies of three full-rank states $\varrho, \varrho^{\prime}$ and $\varrho^{\prime \prime}$ one has

$$
\begin{aligned}
D\left(\varrho \| \varrho^{\prime \prime}\right)-D\left(\varrho \| \varrho^{\prime}\right) & -D\left(\varrho^{\prime} \| \varrho^{\prime \prime}\right)=D\left(\varrho \| \varrho^{\prime \prime}\right)-D\left(\varrho \| \varrho^{\prime}\right)-D\left(\varrho^{\prime} \| \varrho^{\prime \prime}\right)+D\left(\varrho^{\prime} \| \varrho^{\prime}\right) \\
& =\frac{1}{\ln (2)}\left[\phi(\boldsymbol{\eta})+\psi\left(\boldsymbol{\theta}^{\prime \prime}\right)-\boldsymbol{\eta} \cdot \boldsymbol{\theta}^{\prime \prime}-\phi(\boldsymbol{\eta})-\psi\left(\boldsymbol{\theta}^{\prime}\right)+\boldsymbol{\eta} \cdot \boldsymbol{\theta}^{\prime}\right. \\
& \left.-\phi\left(\boldsymbol{\eta}^{\prime}\right)-\psi\left(\boldsymbol{\theta}^{\prime \prime}\right)+\boldsymbol{\eta}^{\prime} \cdot \boldsymbol{\theta}^{\prime \prime}+\phi\left(\boldsymbol{\eta}^{\prime}\right)+\psi\left(\boldsymbol{\theta}^{\prime}\right)-\boldsymbol{\eta}^{\prime} \cdot \boldsymbol{\theta}^{\prime}\right] \\
& =\frac{1}{\ln (2)}\left(\boldsymbol{\eta}-\boldsymbol{\eta}^{\prime}\right) \cdot\left(\boldsymbol{\theta}^{\prime}-\boldsymbol{\theta}^{\prime \prime}\right)
\end{aligned}
$$

and thus

$$
D\left(\varrho \| \varrho^{\prime \prime}\right)=D\left(\varrho \| \varrho^{\prime}\right)+D\left(\varrho^{\prime} \| \varrho^{\prime \prime}\right)+\frac{1}{\ln (2)}\left(\boldsymbol{\eta}-\boldsymbol{\eta}^{\prime}\right) \cdot\left(\boldsymbol{\theta}^{\prime}-\boldsymbol{\theta}^{\prime \prime}\right)
$$

$\ddagger$ Note that the binary logarithm is used in our definition of $D(\varrho \| \chi)$. 
If the scalar product vanishes, this relation is also called the generalized Pythagoras theorem [16].

\subsection{Exponential families and the information projection}

We next define the exponential families of states generated by $k$-particle Hamiltonians, objects of this type will be the focus of the work presented here. For a given multi-index $\alpha=\left(\alpha_{1}, \ldots, \alpha_{n}\right)$ we write $W(\alpha)$ for the weight of $\alpha$, i.e., the number of factors in the Pauli operator $\sigma_{\alpha}=\sigma_{\alpha_{1}} \otimes \cdots \otimes \sigma_{\alpha_{n}}$ different from the identity. In other words the weight $W(\alpha)$ of a multi-index $\alpha$ is the number of nonzero elements $\alpha_{i}$.

For any $1 \leq k \leq n$ we then can define the so-called exponential family $\mathcal{Q}_{k}$ of thermal states of $k$-party Hamiltonians as

$$
\mathcal{Q}_{k}=\left\{\varrho \mid \varrho=\exp \left(\sum_{\alpha: W(\alpha) \leq k} \theta_{\alpha} \sigma_{\alpha}\right)\right\},
$$

so that $\mathcal{Q}_{k}$ is the set of all quantum states, for which the exponential representation contains only $k$-body interactions in the Hamiltonian. Since the exponential representation is unique and the operators $\sigma_{\alpha}$ form a basis of the operator space, this definition is unambiguous. It will also be useful to write $\mathcal{H}_{k}$ for the space of all Hamiltonians containing only interaction terms up to weight $k$.

The set $\mathcal{Q}_{k}$ represents a manifold in the space of all quantum states (see also Fig. 1), a direct characterization is not straightforward. Obviously, one has $\mathcal{Q}_{k} \subset \mathcal{Q}_{k+1}$ and consequently the exponential families define a hierarchy

$$
\mathcal{Q}_{1} \subset \mathcal{Q}_{2} \subset \cdots \subset \mathcal{Q}_{n}
$$

where $\mathcal{Q}_{n}$ is the set of all states with full rank and $\mathcal{Q}_{1}$ the set of all product states with full rank. For states $\varrho$ with full rank we will then ask what the minimal order of interaction, $k$, is such that $\varrho \in \mathcal{Q}_{k}$. For states which are not of full rank, the analogous question is whether the state is in the closure $\overline{\mathcal{Q}}_{k}$ of an exponential family. The introduction of the closure of exponential families makes the discussions and results that follow applicable to quantum states for which some of the eigenvalues vanish.

For a given state quantum state $\varrho$ one can then construct the distance from the exponential family $\mathcal{Q}_{k}$, and in particular the state in $\mathcal{Q}_{k}$ which is the closest to $\varrho$. This defines the so-called information projection:

Definition 1. The information projection $\tilde{\varrho}_{k}$ of a quantum state $\varrho$ is the element of the exponential family $\mathcal{Q}_{k}$ which is the closest to $\varrho$ with respect to the quantum relative entropy,

$$
\tilde{\varrho}_{k}=\operatorname{argmin}_{\varrho^{\prime} \in \mathcal{Q}_{k}} D\left(\varrho \| \varrho^{\prime}\right),
$$

where $D(\varrho \| \chi)=\operatorname{tr}\left[\varrho \log _{2}(\varrho)\right]-\operatorname{tr}\left[\varrho \log _{2}(\chi)\right]$. The distance to the information projection is then considered as a complexity or correlation measure and is given by

$$
D_{k}(\varrho)=\inf _{\varrho^{\prime} \in \mathcal{Q}_{k}} D\left(\varrho \| \varrho^{\prime}\right)=D\left(\varrho \| \tilde{\varrho}_{k}\right)
$$




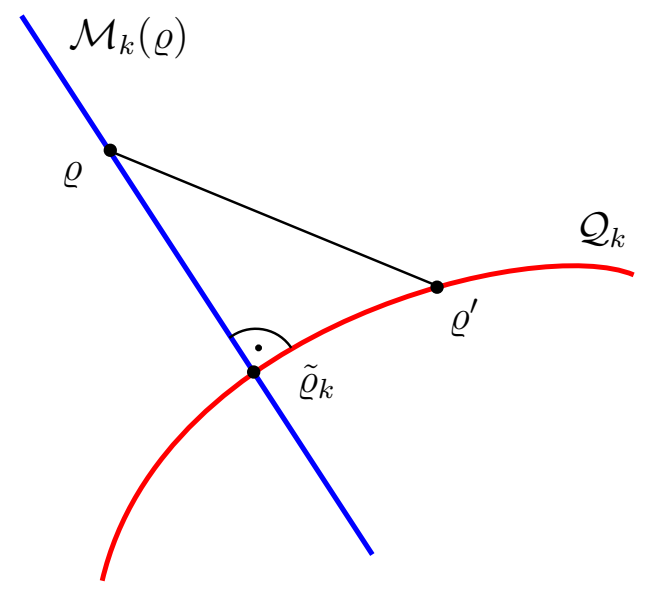

Figure 1. Illustration of the information projection onto a quantum exponential family. Shown are the linear family $\mathcal{M}_{k}(\varrho)$ of distributions with the same $k$-party reduced density matrices as $\varrho$ (blue line), the exponential family $\mathcal{Q}_{k}$ of thermal states of $k$-party Hamiltonians (red curve) and the information projection $\tilde{\varrho}_{k}$ of $\varrho$ onto $\mathcal{Q}_{k}$; and $\varrho^{\prime}$ represents an arbitrary state in $\mathcal{Q}_{k}$. See text for further details.

In order to carry out the computation of this distance it is useful to have different characterizations of the information projection $\tilde{\varrho}_{k}$. To this end, consider a given state $\varrho$ and define the set $\mathcal{M}_{k}(\varrho)$ of states with the same $k$-party reduced density matrices as $\varrho$

$$
\mathcal{M}_{k}(\varrho)=\left\{\varrho^{\prime} \mid \varrho_{A}^{\prime}=\varrho_{A} \text { for all } A \subseteq\{1, \ldots, n\} \text { with }|A|=k\right\},
$$

where $\varrho_{A}=\operatorname{tr}_{\left\{i_{1}, \ldots, i_{n}\right\} \backslash A}(\varrho)$ is the density matrix which is obtained from $\varrho$ by tracing out all qubits except those with indices in $A$. We note that $\mathcal{M}_{k}(\varrho)$ is a linear subspace of the space of all $n$-qubit density matrices, as opposed to the exponential families $\mathcal{Q}_{k}$. Alternatively, one can also write

$$
\mathcal{M}_{k}\left[\varrho_{\text {aff }}(\boldsymbol{\eta})\right]=\left\{\varrho_{\text {aff }}\left(\boldsymbol{\eta}^{\prime}\right) \mid \eta_{\alpha}^{\prime}=\eta_{\alpha} \text { for all } \alpha \text { with } W(\alpha) \leq k\right\} .
$$

The following Lemma was first proven in Ref. [14] and presents three equivalent constructions of the information projection. It also shows that the state $\tilde{\varrho}_{k}$ in Definition 1 is unique.

Lemma 2. The following conditions on a quantum state $\tilde{\varrho}_{k}$ are equivalent [14]:

(a) The state $\tilde{\varrho}_{k}$ is the information projection in the sense of Definition 1.

(b) The state $\tilde{\varrho}_{k}$ is the maximizer of the von Neumann entropy in the set $\mathcal{M}_{k}(\varrho)$ of all states with the same $k$-party reduced density matrices as $\varrho$,

$$
\tilde{\varrho}_{k}=\underset{\varrho^{\prime} \in \mathcal{M}_{k}(\varrho)}{\operatorname{argmax}} S\left(\varrho^{\prime}\right) \text {. }
$$

(c) The state $\tilde{\varrho}_{k}$ is the unique element of the intersection of the exponential family $\mathcal{Q}_{k}$ with the set $\mathcal{M}_{k}(\varrho)$ of all states sharing the same $k$-party reduced density matrices as $\varrho$

$$
\left\{\tilde{\varrho}_{k}\right\}=\mathcal{Q}_{k} \cap \mathcal{M}_{k}(\varrho) .
$$


The situation is illustrated in Fig. 1. Instead of computing $\tilde{\varrho}_{k}$ by minimizing the distance from $\mathcal{Q}_{k}$, one can also look at the intersection of $\mathcal{Q}_{k}$ with $\mathcal{M}_{k}(\varrho)$, or indeed maximize the entropy among elements of the linear family $\mathcal{M}_{k}(\varrho)$.

Proof. Let us start with the case (b), and show that the characterisation (b) is equivalent to that of (a). The linear family $\mathcal{M}_{k}(\varrho)$ is defined as the set of all states $\varrho^{\prime}$ with the same $k$-particle reduced density matrices. This condition is equivalent to the requirement that $\operatorname{tr}\left(\varrho^{\prime} \sigma_{\alpha}\right)=\lambda_{\alpha}=\operatorname{tr}\left(\varrho \sigma_{\alpha}\right)$ for all $\alpha$ with $W(\alpha) \leq k$. Now, maximizing the entropy under the constraint of given expectation values is a well discussed problem in statistical mechanics [21]. The following results are known: The state maximizing the entropy is of the form $\tilde{\varrho}_{k} \sim \exp \left\{\sum_{\alpha: W(\alpha) \leq k} \theta_{\alpha} \sigma_{\alpha}\right\}$ and the maximum is unique. So it is clear that the maximization in condition (b) results in a unique state $\tilde{\varrho}_{k}$ in $\mathcal{Q}_{k}$, we only have to show that minimizes the relative entropy.

In order to show that, consider a third state $\varrho^{\prime}$ in $\mathcal{Q}_{k}$ (see also Fig. 1). We apply Eq. (10) to the state $\varrho=\varrho_{\text {aff }}(\boldsymbol{\eta})$, the state $\varrho_{k}=\varrho_{\text {aff }}(\tilde{\boldsymbol{\eta}})=\varrho_{\exp }(\tilde{\boldsymbol{\theta}})$ [defined via Eq. (17)] and the states $\varrho^{\prime}=\varrho_{\exp }\left(\boldsymbol{\theta}^{\prime}\right)$ in $\mathcal{Q}_{k}$, resulting in

$$
D\left(\varrho \| \varrho^{\prime}\right)=D\left(\varrho \| \varrho_{k}\right)+D\left(\tilde{\varrho}_{k} \| \varrho^{\prime}\right)+\frac{1}{\ln (2)}(\boldsymbol{\eta}-\tilde{\boldsymbol{\eta}}) \cdot\left(\tilde{\boldsymbol{\theta}}-\boldsymbol{\theta}^{\prime}\right) \text {. }
$$

The terms in the scalar product with $W(\alpha) \leq k$ vanish, as we have $\tilde{\varrho}_{k} \in \mathcal{M}_{k}(\varrho)$ and thus $\eta_{\alpha}=\tilde{\eta}_{\alpha}$ for these $\alpha$. The terms with the terms with $W(\alpha)>k$ vanish because of $\tilde{\theta}_{\alpha}=\theta_{\alpha}^{\prime}=0$. So one has $D\left(\varrho \| \varrho^{\prime}\right)=D\left(\varrho \| \tilde{\varrho}_{k}\right)+D\left(\tilde{\varrho}_{k} \| \varrho^{\prime}\right)$, which implies that $\tilde{\varrho}_{k}$, as defined in Eq. (17), is also the unique state minimizing the relative entropy $D\left(\varrho \| \varrho^{\prime}\right)$ among all $\varrho^{\prime} \in \mathcal{Q}_{k}$.

Let us now turn to the characterisation (c). The state defined in (b) is obviously in the intersection $\mathcal{Q}_{k} \cap \mathcal{M}_{k}(\varrho)$, so all we have to show is that this intersection consists of only a single state. Let us assume the contrary, so that there are two states $\varrho_{1}$ and $\varrho_{2}$ in $\mathcal{Q}_{k} \cap \mathcal{M}_{k}(\varrho)$. Then, applying Eq. (10) and the same argument as above one finds that $0=D\left(\varrho_{1} \| \varrho_{1}\right)=D\left(\varrho_{1} \| \varrho_{2}\right)+D\left(\varrho_{2} \| \varrho_{1}\right)$. Since the relative entropy is positive semidefinite, this implies that $\varrho_{1}=\varrho_{2}$.

\subsection{Complexity measures: Definitions and Properties}

As already mentioned, a central topic of this paper is the computation of the distance $D_{k}(\varrho)$ as defined in Eq. (14). Before presenting our results, it is useful to collect some of the properties of the distance measure $D_{k}(\cdot)$. First, note that $D_{k}$ can increase under local transformations, if $k \geq 2[14,15]$. This means that $D_{k}$ cannot in a naive way be viewed as a correlation measure, and so we prefer to call it a complexity measure.

Next one can define the degree of irreducible $k$-party interaction as

$$
C_{k}(\varrho)=D_{k-1}(\varrho)-D_{k}(\varrho), \quad k=2, \ldots, n
$$

(where $D_{n} \equiv 0$ ). The quantity $C_{k}(\varrho)$ describes the extent to which the approximation of a state, $\varrho$, improves, if the allowed interactions in a Hamiltonian increase from $(k-1)$ body interactions to $k$-body interactions. By the generalized Pythagoras theorem, the 
last definition is directly equivalent to

$$
C_{k}(\varrho)=D\left(\tilde{\varrho}_{k} \| \tilde{\varrho}_{k-1}\right), \quad k=2, \ldots, n-1 .
$$

Furthermore, writing $\varrho=\varrho_{\text {aff }}(\boldsymbol{\eta})$ and $\varrho_{k}=\varrho_{\text {aff }}(\tilde{\boldsymbol{\eta}})=\varrho_{\exp }(\tilde{\boldsymbol{\theta}})$, we have, using Eqs. (7) and (8),

$$
\begin{aligned}
\ln (2) D_{k}(\varrho) & =\ln (2) D\left(\varrho \| \varrho_{k}\right)=-\ln (2) S(\varrho)+\psi(\tilde{\boldsymbol{\theta}})-\boldsymbol{\eta} \cdot \tilde{\boldsymbol{\theta}} \\
& =-\ln (2) S(\varrho)+\psi(\tilde{\boldsymbol{\theta}})-\tilde{\boldsymbol{\eta}} \cdot \tilde{\boldsymbol{\theta}} \\
& =-\ln (2) S(\varrho)+\ln (2) S\left(\tilde{\varrho}_{k}\right) .
\end{aligned}
$$

This shows that

$$
D_{k}(\varrho)=S\left(\tilde{\varrho}_{k}\right)-S(\varrho), \quad k=1, \ldots, n-1,
$$

and consequently

$$
C_{k}(\varrho)=S\left(\tilde{\varrho}_{k-1}\right)-S\left(\tilde{\varrho}_{k}\right), \quad k=2, \ldots, n-1 .
$$

These different expressions for $D_{k}$ and $C_{k}$ can be useful for investigating the performance of numerical algorithms to compute the information projection: Having obtained the projections $\tilde{\varrho}_{k}$, one can compute the distances $D_{k}(\varrho)=D\left(\varrho \| \varrho_{k}\right)$ and the interaction measures $C_{k}(\varrho)$. The latter can be calculated in three different ways, namely via Eq. (20), Eq. (21) or Eq. (24). If $\tilde{\varrho}_{k}$ is not the correct information projection, these three expressions will in general give different values.

Finally, let us discuss the case $k=1$ in some more detail. The quantity $D_{1}$ is also referred to as the multi-information [20] or the degree of total interaction. It has an expansion into a telescopic sum of entropy differences

$$
C_{\text {tot }}(\varrho)=D_{1}(\varrho)=\sum_{k=2}^{n} C_{k}(\varrho) .
$$

This is an orthogonal decomposition in the sense of the generalized Pythagoras theorem.

The exponential family $\mathcal{Q}_{1}$ consists of all product states (with full rank). The projection of a state $\varrho$ onto this family is given by the tensor product of the one-party reduced density matrices,

$$
\tilde{\varrho}_{1}=\varrho_{\{1\}} \otimes \cdots \otimes \varrho_{\{n\}} \quad \text { where } \quad \varrho_{\{i\}}=\operatorname{tr}_{\{1, \ldots, n\} \backslash\{i\}} \varrho .
$$

For the other projections there is no such explicit formula. Moreover, the family $\mathcal{Q}_{1}$ is invariant under local filtering transformations of the form

$$
\varrho \mapsto \sigma=\left[F_{1} \otimes F_{2} \otimes \ldots \otimes F_{n}\right] \varrho\left[F_{1}^{\dagger} \otimes F_{2}^{\dagger} \otimes \ldots \otimes F_{n}^{\dagger}\right]
$$

where the $F_{i}$ are arbitrary matrices, since these transformations preserve the product structure [26]. This means that the quantity $D_{1}$ cannot increase under these transformations either [15]. The exponential families $\mathcal{Q}_{k}$ with $k \geq 2$ do not have this property. 


\section{Symmetries}

The computation of the quantities $D_{k}(\varrho)$ and $C_{k}(\varrho)$ is not straightforward. One may therefore ask, whether symmetries of the state $\varrho$ can simplify the optimization procedure. For instance, if $\varrho$ is invariant under the permutation of the first two particles, it seems natural that the state $\tau \in \mathcal{Q}_{k}$ (and the corresponding $k$-party Hamiltonian) which minimize $D_{k}(\varrho)$ share the same permutation symmetry. This symmetry assumption seems plausible, and the following Lemma presents a rigorous statement:

Lemma 3. Let $\varrho$ be a quantum state which has a symmetry of the form

$$
\varrho=U \varrho U^{\dagger}
$$

where $U$ is a unitary matrix that keeps the set of all $k$-particle Hamiltonians invariant, i.e. it is a transformation so that the transformed operator $U H_{k} U^{\dagger}$ is again a $k$-particle Hamiltonian for any $k$-particle operator $H_{k}$. Then the state $\tilde{\varrho}_{k} \in \mathcal{Q}_{k}$ as well as the Hamiltonian $H_{k}$ minimizing the distance $D(\varrho \| \tau)$ have the same symmetry as $\varrho$. This means that one can restrict the optimization to states and Hamiltonians which fulfil the condition

$$
\tau=U \tau U^{\dagger} \quad \text { and } \quad H_{k}=U H_{k} U^{\dagger}
$$

respectively.

Proof. Consider a given $\varrho$ with the symmetry and let $H \in \mathcal{H}_{k}$ be the Hamiltonian of the state $\tau \in \mathcal{Q}_{k}$ minimizing $D(\varrho \| \tau)$. Here, $H$ denotes only the terms which are not proportional to the identity in the Hamiltonian, i.e. one has $\tau=\exp (H) \exp [-\psi(H)]$, where $\exp [-\psi(H)]$ is the normalization [see Eq. (3) $]$. The idea is to consider $H^{\prime}=$ $\left(H+U H U^{\dagger}\right) / 2$ and the corresponding $\tau^{\prime}=\exp \left(H^{\prime}\right)$ and to prove that $D\left(\varrho \| \tau^{\prime}\right) \leq D(\varrho \| \tau)$. From the conditions on $U$ it then follows that $H^{\prime} \in \mathcal{H}_{k}$ and the uniqueness of the information projection implies that $H=H^{\prime}$ and therefore $H=U H U^{\dagger}$.

From Eq. (6) on sees that $D(\varrho \| \tau)$ and $D\left(\varrho \| \tau^{\prime}\right)$ differ only in the contributions from the normalizations $\psi(H)$ [or $\left.\psi\left(H^{\prime}\right)\right]$. In fact, $D\left(\varrho \| \tau^{\prime}\right) \leq D(\varrho \| \tau)$ is equivalent to

$$
\psi\left(H^{\prime}\right)=\ln \left\{\operatorname{tr}\left[\exp \left(H^{\prime}\right)\right]\right\} \leq \psi(H)=\ln \{\operatorname{tr}[\exp (H)]\} .
$$

So it suffices to show $\operatorname{tr}\left[\exp \left(A+U A U^{\dagger}\right)\right] \leq \operatorname{tr}[\exp (2 A)]$ for arbitrary hermitean matrices $A$. Applying the Golden-Thompson inequality $\operatorname{tr}[\exp (A+B)] \leq \operatorname{tr}[\exp (A) \exp (B)](\operatorname{see}$ page 261 in Ref. [27]) we have $\operatorname{tr}\left[\exp \left(A+U A U^{\dagger}\right)\right] \leq \operatorname{tr}\left[\exp (A) U \exp (A) U^{\dagger}\right]$ and it remains to show that $\operatorname{tr}\left(X U X U^{\dagger}\right) \leq \operatorname{tr}\left(X^{2}\right)$ for $X=\exp (A)$. Taking the spectral decomposition $X=\sum_{k} \lambda_{k}\left|\phi_{k}\right\rangle\left\langle\phi_{k}\right|$ this reads $\sum_{k l} C_{k l} \lambda_{k} \lambda_{l} \leq \sum_{k} \lambda_{k}^{2}$, where $C_{k l}=\left|\left\langle\phi_{k}|U| \phi_{l}\right\rangle\right|^{2}$ is a doubly stochastic matrix, that is, the row sums and column sums of $C$ equal one. Birkhoff's Theorem states that any doubly stochastic matrix can always be written as a convex combination of permutation matrices, $C=\sum_{k} p_{k} \Pi_{k}$, where the $p_{k}$ form a probability distribution (see page 527 in Ref. [28]). So it remains to show that $\sum_{k} \lambda_{k} \lambda_{\pi(k)} \leq \sum_{k} \lambda_{k}^{2}$ for an arbitrary permutation $\pi$, but this follows directly from the Cauchy Schwartz inequality.

This Lemma can be used in various situations to simplify the calculation of the information projection: 
- Permutation symmetry: If $\varrho$ is invariant under permutation of the particles $i$ and $j$, then this is a unitary symmetry as in Lemma 3, with the unitary flip operator $U=F_{i j}$. This unitary operation also fulfils the other conditions of Lemma 3, and one can conclude that it suffices to optimize over Hamiltonians with the same permutation symmetry. Exploiting this symmetry can reduce the number of parameters in numerical algorithms significantly.

- Graph state symmetry: In the framework of quantum information theory, so-called graph states and stabilizer operators have attracted significant attention [29]. They are defined as follows: Consider an $n$-qubit system, and $n$ observables $g_{i}$ which are tensor products of Pauli matrices and which commute pairwise. For example, for three qubits one can take $g_{1}=\sigma_{z} \otimes \sigma_{z} \otimes \mathbb{1}, g_{2}=\mathbb{1} \otimes \sigma_{z} \otimes \sigma_{z}$, and $g_{3}=\sigma_{x} \otimes \sigma_{x} \otimes \sigma_{x}$. One can further consider all products of the $g_{i}$. This is an the Abelian group with $2^{n}$ elements ( since $g_{i}^{2}=\mathbb{1}$ ) and this group is called the stabilizer. One can alternatively characterize the stabilizer as all tensor products of Pauli matrices, which commute with all $g_{i}$.

A graph state $|G\rangle$ is defined as an eigenstate of all $g_{i}$ with eigenvalue +1 , that is $g_{i}|G\rangle=|G\rangle$. For the three-qubit example above, the graph state is given by the well-known GHZ state $|G H Z\rangle=(|000\rangle+|111\rangle) / \sqrt{2}$. Allowing also \pm 1 eigenvalues one obtains a basis of $2^{n}$ graph states $\left|G_{k}\right\rangle$.

A quantum state diagonal in the basis of the $\left|G_{k}\right\rangle$ is a graph-diagonal state, and such states have been intensively studied [30,31. These states fulfil

$$
\varrho_{\mathrm{GD}}=g_{i}\left(\varrho_{\mathrm{GD}}\right) g_{i}^{\dagger}
$$

and since the $g_{i}=g_{i}^{\dagger}$ are unitary, Lemma 3 can be applied. One can directly see that the only possible $k$-particle interaction terms in the Hamiltonian which share the same symmetry are just all terms from the stabilizer group which are of weight $k$ or less. For small $k$ these are typically very few terms, which simplifies calculations significantly. Note that this structure was also observed for special graph-diagonal states in Ref. [13], but Lemma 3 shows that this holds for all mixed graph-diagonal states.

- $U^{\otimes n}$-symmetry: Another family of states where Lemma 3 can be applied are the so-called $U^{\otimes n}$-invariant states. These states fulfil

$$
\varrho=U^{\otimes n} \varrho\left(U^{\dagger}\right)^{\otimes n}
$$

for all possible unitary transformations $U$ on a single particle. For two particles these states are the Werner states [32, but also for more particles detailed characterizations are known [33,34]. Lemma 3 shows that when computing $D_{k}(\varrho)$ for these states, the optimal Hamiltonian has the same $U^{\otimes n}$-symmetry. This also implies that the optimal Hamiltonian has no single-particle terms, since the only single-qubit operator with this symmetry is the identity. It follows that for arbitrary $U^{\otimes n}$-invariant states the multiinformation is simply given by

$$
D_{1}(\varrho)=D_{0}(\varrho)=n-S(\varrho),
$$


where $D_{0}(\varrho)$ denotes the distance to the maximally mixed state.

\section{Iterative computation of the quantum information projection}

In this section we present an algorithm for computing the information projection. Other existing algorithms will be discussed in Section 5 .

\subsection{Preliminary considerations}

In this section we will describe an efficient numerical algorithm with which to compute the projection $\tilde{\varrho}_{k}$ of a given $n$-particle quantum state $\varrho$ on an exponential family $\mathcal{Q}_{k}$. To this end one needs to construct $\tilde{\varrho}_{k} \in \mathcal{Q}_{k}$ such that $\tilde{\varrho}_{k} \in M_{k}(\varrho)$, i.e. such that $\varrho_{k}$ has the same $k$-particle reduced density matrices as $\varrho$. The algorithm we put forward is in spirit- similar to the iterative projection algorithm for the classical case proposed in Refs. [17, 18. We start with the fully mixed state $\varrho^{\prime}=\mathbb{1} / \operatorname{tr}(\mathbb{1})$ as an approximation for $\tilde{\varrho}_{k}$, at each iteration step of the algorithm the current approximation of $\tilde{\varrho}_{k}$ is then improved such as to better match the reduced density matrix of $\varrho$ defined by a particular subset of particles. The algorithm proceeds by iteratively going through all such subsets of at most $k$ particles repeatedly until convergence is reached.

Specifically, let $\varrho$ be the state whose projection onto $\mathcal{Q}_{k}$ we want to calculate, and let $\tau=\mathrm{e}^{H} / \operatorname{tr}\left(\mathrm{e}^{H}\right)$ be the current approximation of $\tilde{\rho}_{k}$, where $H$ is a $k$-party Hamiltonian. The algorithm is initiated from $H=\mathbb{1}$.

For a fixed $\ell$-party observable $A$ with $\ell \leq k$ the iteration step consists of adding a term $\varepsilon A$ to the Hamiltonian $H$, generating an updated approximation $\tau^{\prime}$ of $\varrho$. The amplitude of the modification, $\varepsilon$, is chosen such that the expectation of $A$ under the density matrix $\tau^{\prime}$ improves the match with the expectation obtained under $\varrho$. In detail one has the update

$$
\tau=\frac{\mathrm{e}^{H}}{\operatorname{tr}\left(\mathrm{e}^{H}\right)} \rightarrow \tau^{\prime}(\varepsilon)=\frac{\mathrm{e}^{H+\varepsilon A}}{\operatorname{tr}\left(\mathrm{e}^{H+\varepsilon A}\right)},
$$

such that $\operatorname{tr}\left(A \tau^{\prime}\right)$ matches $\operatorname{tr}(A \varrho)$ as closely as possible. In principle $\varepsilon$ can be obtained by solving $\operatorname{tr}\left(A \tau^{\prime}\right)=\operatorname{tr}(A \varrho)$ directly. In practice this is hard to implement though, as $\operatorname{tr}\left(A \tau^{\prime}\right)$ depends non-linearly on $\varepsilon$. We therefore resort to the following linear approximation

where one has

$$
\operatorname{tr}\left[A \tau^{\prime}(\varepsilon)\right]=\operatorname{tr}(A \tau)+\left.\varepsilon \frac{\partial}{\partial \varepsilon} \operatorname{tr}\left[A \tau^{\prime}(\varepsilon)\right]\right|_{\varepsilon=0}+\mathcal{O}\left(\varepsilon^{2}\right),
$$

$$
\frac{\partial}{\partial \varepsilon} \operatorname{tr}\left[A \tau^{\prime}\right]=\operatorname{tr}\left[A \frac{\partial_{\varepsilon} \mathrm{e}^{H+\varepsilon A}}{\operatorname{tr}\left(\mathrm{e}^{H+\varepsilon A}\right)}\right]-\operatorname{tr}\left[A \frac{\mathrm{e}^{H+\varepsilon A}}{\operatorname{tr}\left(\mathrm{e}^{H+\varepsilon A}\right)}\right] \frac{\operatorname{tr}\left(\partial_{\varepsilon} \mathrm{e}^{H+\varepsilon A}\right)}{\operatorname{tr}\left(\mathrm{e}^{H+\varepsilon A}\right)} .
$$

We have here used the shorthand notation $\partial_{\varepsilon}=\frac{\partial}{\partial \varepsilon}$. When evaluating the derivative of the matrix exponential in this expression one has to take into account that $H$ and $A$ generally do not commute. So it is convenient to use the identity [35]

$$
\frac{\partial}{\partial t} e^{M(t)}=\int_{0}^{1} d s e^{s M(t)} \frac{\partial M(t)}{\partial t} e^{-s M(t)} e^{M(t)},
$$


valid for a general one-parameter family of matrices $M(t)$. Applying this identity to substitute for the derivatives in Eq. (36) and carrying out a modest amount of algebra one obtains

$$
\left.\frac{\partial}{\partial \varepsilon} \operatorname{tr}\left[A \tau^{\prime}(\varepsilon)\right]\right|_{\varepsilon=0}=\frac{1}{\operatorname{tr}\left(\mathrm{e}^{H}\right)} \int_{0}^{1} d s \operatorname{tr}\left[A \mathrm{e}^{s H} A \mathrm{e}^{-s H} \mathrm{e}^{H}\right]-[\operatorname{tr}(A \tau)]^{2} .
$$

Next we apply a further approximation to the remaining integral, and replace it by the mean of the integrand evaluated at the upper and lower limits $s=0$ and $s=1$, respectively. This gives

$$
\int_{0}^{1} d s \operatorname{tr}\left(A \mathrm{e}^{s H} A \mathrm{e}^{-s H} \mathrm{e}^{H}\right) \approx \frac{1}{2}\left[\operatorname{tr}\left(A \mathrm{e}^{H} A\right)+\operatorname{tr}\left(A^{2} e^{H}\right)\right]=\operatorname{tr}\left(A^{2} \mathrm{e}^{H}\right) .
$$

This in turn leads to

$$
\operatorname{tr}\left[A \tau^{\prime}(\varepsilon)\right] \approx \operatorname{tr}(A \tau)+\varepsilon\left\{\operatorname{tr}\left(A^{2} \tau\right)-[\operatorname{tr}(A \tau)]^{2}\right\}
$$

Admittedly, this approximation can only be justified a posteriori by the performance of the algorithm. Setting $\operatorname{tr}\left[A \tau^{\prime}\right]=\operatorname{tr}[A \varrho]$ and using the approximation just obtained one finds the following solution for $\varepsilon$ :

$$
\varepsilon \approx \frac{\operatorname{tr}(A \varrho)-\operatorname{tr}(A \tau)}{\operatorname{tr}\left(A^{2} \tau\right)-[\operatorname{tr}(A \tau)]^{2}}=\frac{\langle A\rangle_{\varrho}-\langle A\rangle_{\tau}}{\Delta_{\tau}^{2}(A)}
$$

where we have used the notation $\langle A\rangle_{\varrho}=\operatorname{tr}[A \varrho]$ (and analogously for $\langle A\rangle_{\tau}$ ), and where $\Delta_{\tau}^{2}(A)=\left\langle A^{2}\right\rangle_{\tau}-\langle A\rangle_{\tau}^{2}$ is the variance.

\subsection{Description of the algorithm}

In the full algorithm with which to compute the information projection of $\varrho$ onto the quantum exponential family $\mathcal{Q}_{k}$, one chooses an orthogonal basis $V_{k}$ in the space of $k$-party observables (excluding the identity) and updates the approximation $\tau$ for each $A \in V_{k}$ in turn. For an $n$-qubit system one can choose the Pauli operators

$$
V_{k}=\left\{\tau_{\alpha} \mid 1 \leq W(\alpha) \leq k\right\} .
$$

The complete algorithm is then as follows:

Problem: Given an $n$-qubit state $\varrho$, compute its information projection $\tilde{\varrho}_{k}$ onto the exponential family $\mathcal{Q}_{k}$.

Algorithm:

1. Choose an orthonormal basis $V_{k}$ of the space of $k$-party observables, say these observables are $A_{1}, A_{2}, \ldots, A_{M}$ (where $M$ will depend on $k$ ). For each element $A_{i} \in V_{k}$ compute the expectation value $\left\langle A_{i}\right\rangle_{\varrho}$.

2. Initialize $\tau=\mathbb{1} / 2^{n}$ as the completely mixed state.

3. (a) Start with $i=1$, and update $\tau$ according to

$$
\tau=\frac{\mathrm{e}^{H}}{\operatorname{tr}\left(\mathrm{e}^{H}\right)} \rightarrow \tau^{\prime}=\frac{\mathrm{e}^{H+\varepsilon A_{i}}}{\operatorname{tr}\left(\mathrm{e}^{H+\varepsilon A_{i}}\right)} \quad \text { where } \quad \varepsilon=\frac{\left\langle A_{i}\right\rangle_{\varrho}-\left\langle A_{i}\right\rangle_{\tau}}{\Delta_{\tau}^{2}\left(A_{i}\right)} .
$$


(b) Increment $i$ to $i+1$ and repeat step 3(a). Once the coefficients for all observable in $V_{k}$ have been updated (i.e for $i=1, \ldots, M$ ) goto 4 .

4. If the maximum number of iterations has been reached or a convergence criterion is met, terminate, otherwise goto 3 .

When implementing the algorithm, it turns out to be useful to introduce an additional parameter $\omega$ which controls the size of the steps in the space of Hamiltonians,

$$
\tau=\frac{\mathrm{e}^{H}}{\operatorname{tr}\left(\mathrm{e}^{H}\right)} \rightarrow \tau^{\prime}=\frac{\mathrm{e}^{H+\omega \varepsilon A}}{\operatorname{tr}\left(\mathrm{e}^{H+\omega \varepsilon A}\right)}
$$

with $\varepsilon$ as above. Choosing values $\omega<1$ corresponds to what is known as a successive underrelaxation scheme [36] and it can improve the convergence properties of the algorithm.

We would like to stress that we do not have a proof that the algorithm converges. For the classical case, however, it has been shown that a similar algorithm converges [17, 18]. One could also think of improving our algorithm by using a better approximation to the integral. However, the numerical results shown below demonstrate that the algorithm as described here works remarkably well.

\subsection{Test of the algorithm}

In order to test the algorithm just described, we consider Dicke states of four and six qubits. These states are given by

$$
\begin{aligned}
& \left|D_{2}^{4}\right\rangle=\frac{1}{\sqrt{6}}(|0011\rangle+|0101\rangle+|0110\rangle+|1001\rangle+|1010\rangle+|1100\rangle), \\
& \left|D_{3}^{6}\right\rangle=\frac{1}{\sqrt{20}}(|000111\rangle+\text { permutations }),
\end{aligned}
$$

that is, they are a balanced superposition of all terms in the standard basis with $n / 2$ excitations. Dicke states have intensively been studied in entanglement theory and have been observed in several experiments [3]. As a one-parameter family of states, we consider Dicke states mixed with white noise,

$$
\varrho^{(n)}(p)=p \frac{\mathbb{1}}{2^{n}}+(1-p)\left|D_{n / 2}^{n}\right\rangle\left\langle D_{n / 2}^{n}\right| .
$$

Before applying our algorithm, it is useful to discuss the symmetries of these states. First, the states $\varrho^{(n)}(p)$ are symmetric under the exchange of particles. Consequently, the information projection $\tilde{\varrho}_{k}$ shares the same symmetry. There are, however, additional symmetries: If we consider the operators

$$
G_{x}^{(n)}=\sigma_{x}^{\otimes n} \text { and } G_{z}^{(n)}=\sigma_{z}^{\otimes n}
$$

then it directly follows that $G_{\alpha}^{(n)}\left[\varrho^{(n)}(p)\right]\left(G_{\alpha}^{(n)}\right)^{\dagger}=\varrho^{(n)}(p)$ for $\alpha=x, z$, which implies that the information projection $\tilde{\varrho}_{k}$ and the corresponding minimising Hamiltonians have this symmetry as well. Note that $\varrho^{(n)}(p)$ is also symmetric under the product $G_{y}^{(n)}=G_{x}^{(n)} G_{z}^{(n)}$, but this is not an independent symmetry. 

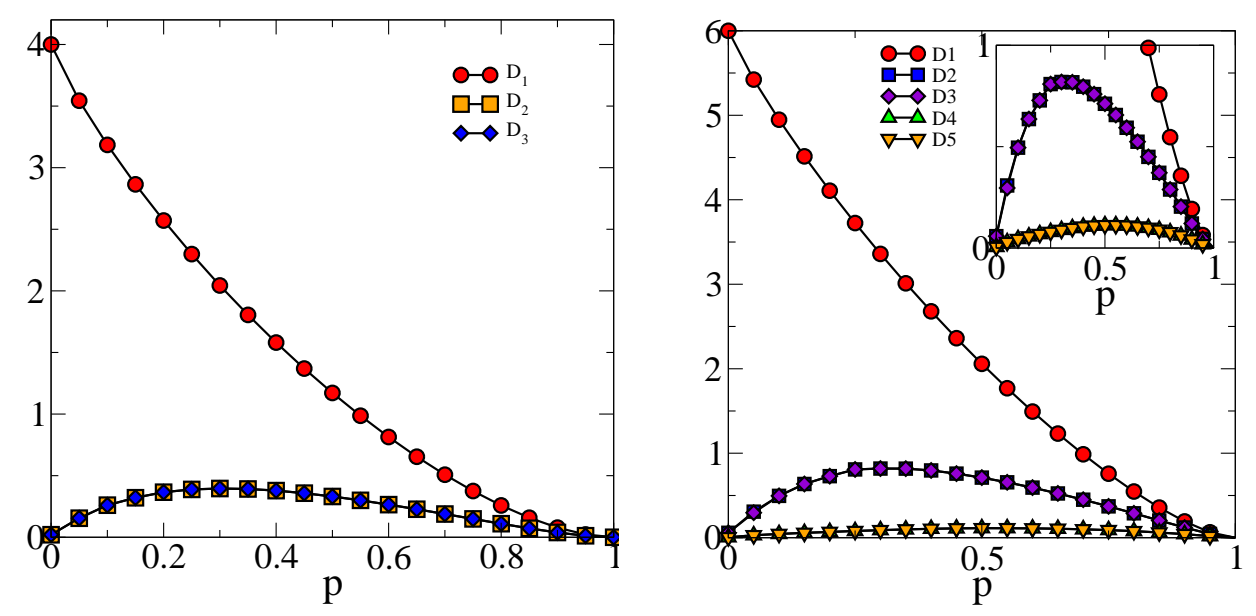

Figure 2. Complexity measures for Dicke states mixed with white noise (left: the four-qubit case, right: the six-qubit case). See text for further details.

These symmetries reduce the number of parameters already significantly. For instance, there are no single-particle Hamiltonians, which are invariant under these symmetries, so we have $D_{1}(\varrho)=D_{0}(\varrho)=n-S(\varrho)$, as for the $U^{\otimes n}$-invariant states discussed above. Similarly many possible interaction terms can be discarded from the outset for higherorder interactions. Applying our algorithm generates the data shown in Fig. 2. We have here chosen an under-relaxation parameter of $\omega=0.5$ for $\varrho^{(4)}(p)\left[\omega=0.1\right.$ for $\left.\varrho^{(6)}(p)\right]$, although $\omega=1$ yields similar results when convergence is reached. We typically run the algorithmic scheme for up to 100 iterations [500 iterations for $\varrho^{(6)}(p)$ ], or until a convergence threshold is met, and we report the minimum distance reached over this number of iterations. It must be stressed though that our numerical results are estimates of the respective distances, we cannot exclude that the precise quantitative results have a remaining dependence on parameters of the algorithm, such as the relaxation parameter $\omega$, the maximum number of iteration steps, or the precise convergence criterion.

For the four-qubit case, one finds that the measures $D_{2}$ and $D_{3}$ coincide. This can be explained as follows: Let us consider the information projection $\tilde{\varrho}_{2}=\exp \left(H_{2}\right)$ and the corresponding two-particle Hamiltonian $H_{2} \in \mathcal{H}_{2}$. As already mentioned above, $H_{2}$ does not contain any single-particle term. For the two-particle terms there are also not many possibilities, in fact, the only possible terms are $h_{\alpha}^{1,2}=\sigma_{\alpha} \otimes \sigma_{\alpha} \otimes \mathbb{1} \otimes \mathbb{1}$ for $\alpha=x, y, z$, and permutations thereof. Using the power series of the exponential function, one finds that $\varrho_{2}=\exp \left(H_{2}\right)$ has no three-body correlations in its Bloch representation, that is, $\operatorname{tr}\left[\left(\sigma_{i} \otimes \sigma_{j} \otimes \sigma_{k} \otimes \mathbb{1}\right) \varrho_{2}\right]=0$ for any choice of $i, j, k \in\{x, y, z\}$. In other words, one

$\S$ The detailed proof is the following: $H_{2}$ and $\varrho_{2}$ obey the symmetry defined by the $G_{\alpha}^{(n)}$, which implies already that most of the three-body correlations in the Bloch representation vanish. The only terms which are not forced to be zero are expectation values of $K^{(3)}=\sigma_{x} \otimes \sigma_{y} \otimes \sigma_{z} \otimes \mathbb{1}$ or permutations thereof. However, if $\exp \left(H_{2}\right)$ is written as a power series, the term $K^{(3)}$ does never occur as a product of the two-qubit terms $h_{\alpha}^{i, j}$. The reason is that if the product of the single-qubit observables in $K^{(3)}$ 
can state that the maximizer of the entropy in the linear family $\mathcal{M}_{2}$ has no three-body correlations in its Bloch representation.

On the other hand, let us consider the three-body reduced state of $\varrho^{(4)}(p)$. This state is given by

$$
\varrho_{123}=p \frac{\mathbb{1}}{2^{3}}+\frac{1-p}{6}(\mathbb{1}-|000\rangle\langle 000|-| 111\rangle\langle 111|)
$$

and one can directly check that this state also has no three-body correlations in its Bloch representation. But this means that the maximizer of the the entropy in the linear family $\mathcal{M}_{2}$ has the same reduced three-particle density matrices as $\varrho^{(4)}(p)$, so it is also an element of the smaller linear family $\mathcal{M}_{3}$. From this $D_{2}\left[\varrho^{(4)}(p)\right]=D_{3}\left[\varrho^{(4)}(p)\right]$ follows.

For the six-qubit case, we find that $D_{2}\left[\varrho^{(6)}(p)\right]=D_{3}\left[\varrho^{(6)}(p)\right]$ and $D_{4}\left[\varrho^{(6)}(p)\right]=$ $D_{5}\left[\varrho^{(6)}(p)\right]$ and this can be understood in the same way. In order show that $D_{2}=D_{3}$ one first finds that the reduced three-particle state of $\varrho^{(6)}(p)$ does not contain any three body correlations, then the argument is the same as for the four-qubit Dicke state. For $D_{4}=D_{5}$ one has to consider the reduced five-particle density matrices. Due to the symmetry, the only relevant correlators are $K_{1}^{(5)}=\sigma_{x} \otimes \sigma_{z} \otimes \sigma_{y} \otimes \sigma_{y} \otimes \sigma_{y} \otimes \mathbb{1}$, $K_{2}^{(5)}=\sigma_{x} \otimes \sigma_{z} \otimes \sigma_{z} \otimes \sigma_{z} \otimes \sigma_{y} \otimes \mathbb{1}$ and $K_{3}^{(5)}=\sigma_{x} \otimes \sigma_{x} \otimes \sigma_{x} \otimes \sigma_{z} \otimes \sigma_{y} \otimes \mathbb{1}$. Their mean values vanish in the state $\varrho^{(6)}(p)$, and then the proof can proceed as before.

Besides these examples, we have tested our algorithm for a variety of four- and fivequbit states, and previous results [13, 19] we easily reproduced. This shows that the algorithm presented above is a useful tool for computing the complexity measure for states up to six qubits.

\section{Other algorithms}

In this section, we will first describe another algorithm, which has been proposed to compute the complexity measure [19]. Then, we will discuss how other methods known in numerical optimization can be used for this problem.

\subsection{The algorithm of Ref. [19]}

In Ref. 19 D.L. Zhou has proposed an algorithm for computing the information projection and has presented examples up to five qubits. The idea of the algorithm is as follows.

First, one considers the information projection $\varrho_{k}$ onto the exponential family $\mathcal{Q}_{k}$ and its $\operatorname{logarithm} \log \left(\tilde{\varrho}_{k}\right)$, which is effectively the generating $k$-particle Hamiltonian. Then, according to Lemma 2, $\tilde{\varrho}_{k}$ obeys the following conditions: (i) First, the mean values of Pauli matrices $\sigma_{\alpha}$ in the state $\tilde{\varrho}_{k}$ equal the mean values in the state $\varrho$, if the weight

is taken, the result is $\left(\sigma_{x}\right) \cdot\left(\sigma_{y}\right) \cdot\left(\sigma_{z}\right) \cdot(\mathbb{1})=i \mathbb{1}$, which is a non-hermitian operator. If an arbitrary product of the $h_{\alpha}^{i, j}$ is considered, and then the product of the single-qubit observables is taken, the result is an hermitean operator, since any Pauli matrix occurs an even number of times in the total product. 
$W(\alpha)$ of $\sigma_{\alpha}$ is smaller or equal $k$. This is nothing but the condition that the reduced $k$-particle states of $\tilde{\varrho}_{k}$ and $\varrho$ are the same. (ii) Second, the mean values of Pauli matrices $\sigma_{\alpha}$ in the Hamiltonian $\log \left(\tilde{\varrho}_{k}\right)$ vanish, if the weight $W(\alpha)$ of $\sigma_{\alpha}$ is larger than $k$. This is the condition that the generating Hamiltonian contains no higher-order interactions than $k$-particle interactions.

These conditions lead to $4^{n}$ equalities for $\tilde{\varrho}_{k}$, and from Lemma 2 it follows that there is a unique solution to all these equalities, which is the desired $\tilde{\varrho}_{k}$. Due to the occurrence of the logarithm, however, the equalities are highly nonlinear, and a direct numerical solution is not straightforward. Therefore, as explicitly stated in Ref. [19], one needs an initial guess of an initial value of $\tilde{\varrho}_{k}$ for solving them. For example, in Ref. [19] curves like the ones in Fig. 2 have been computed iteratively: Initially, one solves the problem if the state is completely noisy $(p=1)$, then one uses the solution as an initial value for solving the nonlinear equations for decreasing $p \mapsto p-\varepsilon$ and so on, until $p=0$ is reached. We stress that no such procedure is needed for our algorithm, then data points shown in Fig. 2 are obtained independently for the different values of $p$.

\subsection{Convex optimization approaches}

The algorithm described in Section 4 searches for an approximation of the information projection by an iteration within the exponential family $\mathcal{Q}_{k}$, which is a highly nonlinear manifold. In Lemma 2 it was established that the information projection $\tilde{\varrho}_{k}$ can also be characterised by a maximization of the von Neumann entropy $S\left(\varrho^{\prime}\right)$ over the linear family $\mathcal{M}_{k}(\varrho)$, given by the density matrices with the same $k$-particle reduces density matrices. The advantage of this formulation is that the problem becomes an instance of convex optimization, namely the minimization of the convex function $-S\left(\varrho^{\prime}\right)$ over the convex set $\mathcal{M}_{k}(\varrho)$. Note that convex optimization problems are well-studied, for an overview see Ref. [37].

From this structure it is clear that no local minima exist, i.e., if $-S\left(\varrho^{\prime}\right) \leq-S(\tau)$ for all $\tau \in \mathcal{M}_{k}(\varrho)$ with $\left\|\varrho^{\prime}-\tau\right\|<\varepsilon$ where $\varepsilon>0$, then $\varrho^{\prime}$ attains the global minimum. This makes a numerical solution particularly tractable. In the language of convex optimization, the problem reads

maximize: $t$

subject to: $S\left(\varrho^{\prime}\right) \geq t$

$$
\begin{aligned}
& \operatorname{tr}\left[\left(\varrho^{\prime}-\varrho\right) \sigma_{\alpha}\right]=0 \text { for all } \alpha \text { with } W(\alpha) \leq k, \text { and } \\
& \varrho^{\prime} \geq 0 .
\end{aligned}
$$

For such problems one can construct algorithms where the optimality of the solution is guaranteed.

A problem which is related to the one discussed here was studied by Teo and coworkers in the context of quantum state estimation theory [38]: They consider the situation where in a quantum experiment the observed frequencies $\mathbf{f}$ of measurement outcomes (described by positive operators $E_{i}$ ) are sampled from a probability distribution $P\left[\varrho^{\prime}\right]$ 
from an unknown quantum state $\varrho^{\prime}$ (that is, the probabilities are computed according to $\left.P_{i}=\operatorname{tr}\left(E_{i} \varrho^{\prime}\right)\right)$. Among the solutions which maximize the $\log$-likelihood $\log \left(L\left(\varrho^{\prime} \mid \mathbf{f}\right)\right)$ [which is proportional to $\left.-D\left(\mathbf{f} \| P\left[\varrho^{\prime}\right]\right)\right]$, they propose to use the state with maximum entropy. For the solution of this problem they introduce an iterative algorithm that eventually approaches

$$
\varrho^{*}=\lim _{\lambda \searrow 0} \underset{\varrho^{\prime}}{\operatorname{argmin}}\left[-\log \left(L\left(\varrho^{\prime} \mid \mathbf{f}\right)\right)-\lambda S\left(\varrho^{\prime}\right)\right]
$$

where the minimization is performed over all $\varrho^{\prime} \geq 0$.

In order to see the relation to our problem, consider a probability distribution $P[\tau]_{i}=\operatorname{tr}\left(E_{i} \tau\right)$, where $E_{i}$ are positive semidefinite operators with $\sum_{i} E_{i}=\mathbb{1}$ and $\operatorname{span}\left\{E_{i}\right\}=\operatorname{span} \mathcal{M}_{k}(\varrho)$. This means that knowledge of the probabilities $P[\tau]$ is equivalent to knowledge of the reduced $k$-particle density matrices. If we now replace $\mathbf{f}$ by $P[\varrho]$, then the optimization in Eq. (49) is indeed closely related to our optimization problem: One can view Eq. (49) as a maximization of the entropy $S\left(\varrho^{\prime}\right)$ where the loglikelihood term [being proportional to $\left.D\left(P[\varrho] \| P\left[\varrho^{\prime}\right]\right)\right]$, serves as a barrier term, forcing $\varrho$ and $\varrho^{\prime}$ to have the same reduced $k$-particle states. Such constructions are known from interior point methods for convex optimization 37.

While the methods presented in this section rely on established numerical algorithms, we observed that computing the information projection by solving a convex optimisation problem actually requires more resources than the algorithm we propose in Sec. 4. This is probably due to the fact, that the algorithm of Sec. 4 exploits the structure of the problem in a better way.

\section{Conclusions}

In summary we have used concepts from information geometry to characterise the complexity of multiparticle quantum states. Specifically, we considered the distance of a given $n$-particle density matrix from the space of all thermal quantum states generated by Hamiltonians with $k$-particle interactions. We have shown how symmetries can be used to simplify the calculation of the resulting complexity measure. Furthermore, we have proposed a new algorithm to compute this measure. This algorithm, we think, is computationally more efficient than existing approaches, and in particular we are able to compute the above complexity measures for selected six-particle states.

There are several follow-on problems requiring further attention. First, the complexity measure is not yet fully understood, and several interesting open questions remain, for example, which are the states with a maximal distance $D_{k}$ from a given exponential family? How is the complexity measure related to known entanglement measures or correlation measures? Second, it would be interesting to study this measure in specific situations. For instance, for a given $n$-particle spin model with two-particle interactions only one may consider the reduced states of some of the particles and ask, whether they still can effectively be described as thermal states of a two-body Hamiltonian, or whether higher-order correlations are present. We expect that this may for example be 
of interest in models of quantum spin chains at or near quantum phase transitions. It is known that entanglement measures can be used to study such critical phenomena, and so a systematic exploration of the complexity measures we have proposed here cannot only help to understand quantum phase transitions better, but also to relate different measures of complexity and correlation.

\section{Acknowledgments}

We thank Tobias Moroder for helpful discussions. This work is supported by the Royal Society (reference JP090467), the EU (Marie Curie CIG 293993/ENFOQI) and the BMBF (Chist-Era Project QUASAR).

\section{References}

[1] M. B. Plenio and S. Virmani, Quant. Inf. Comp. 7, 1 (2007).

[2] R. Horodecki, P. Horodecki, M. Horodecki, and K. Horodecki, Rev. Mod. Phys. 81, 865 (2009).

[3] O. Gühne and G. Tóth, Phys. Rep. 474, 1 (2009).

[4] D. L. Zhou, B. Zeng, Z. Xu, and L. You, Phys. Rev. A 74, 052110 (2006).

[5] K. Modi, A. Brodutch, H. Cable, T. Paterek, and V. Vedral, Rev. Mod. Phys. 84, 1655 (2012).

[6] R. Badii and A. Politi, Complexity: Hierarchical Structures and Scaling in Physics, Cambridge University Press (1999).

[7] K. Lindgren and M. G. Nordahl, Complex Systems 2, 409 (1988).

[8] S. Lloyd, Control Systems, IEEE 217 (2001)

[9] D. P. Feldman and J. P. Crutchfield, A survey of complexity measures, Santa Fe Summer School 1998

[10] D. Feldman and J. P. Crutchfield, Phys. Lett. A 238, 244 (1998).

[11] B. Edmonds, Bibliography of Complexity Measures, available at bruce.edmonds .name/combib/.

[12] T. Kahle, E. Olbrich, J. Jost and N. Ay, Phys. Rev. E 79, 026201 (2009).

[13] D. L. Zhou, Phys. Rev. Lett. 101, 180505 (2008).

[14] D. L. Zhou, Phys. Rev. A 80, 022113 (2009).

[15] T. Galla and O. Gühne, Phys. Rev. E 85, 046209 (2012).

[16] S. Amari, IEEE Trans. Inf. Theory 47, 1701 (2001).

[17] I. Csiszár and P. C. Shields, Found. and Trends in Communications and Inf. Theory 1, 417 (2004).

[18] L. Steiner and T. Kahle Computing Information Projections Iteratively with CIPI, available at github.com/tom111/cipi, (accessed August 2010).

[19] D. L. Zhou, arXiv:0909.3700.

[20] N. Ay and A. Knauf, Kybernetika 42, 517 (2007).

[21] M. Stingl, Statistische Physik, lecture notes at the Universität Münster, available at pauli.uni-muenster.de/tp/fileadmin/lehre/skripte/stingl/spskript.ps.zip.

[22] R. K. P. Zia, E. F. Redish and S. R. McKay, American Journal of Physics 77, 614 (2009).

[23] R. Balian, Du microscopique au macroscopique: cours de physique statistique de l'Ecole polytechnique, Ellipses-Edition Marketing, Paris 1982

[24] G. Kimura, Phys. Lett. A 314, 339 (2003).

[25] M. S. Byrd and N. Khaneja, Phys. Rev. A 68, 062322 (2003).

[26] F. Verstraete, J. Dehaene, and B. De Moor, Phys. Rev. A 68, 012103 (2003).

[27] R. Bhatia, Matrix Analysis, Springer-Verlag (Heidelberg) 1997.

[28] R. Horn and C. Johnson, Matrix Analysis, Cambridge University Press (Cambrigde) 1999.

[29] M. Hein, W. Dür, J. Eisert, R. Raussendorf, M. Van den Nest, and H.-J. Briegel, Entanglement in Graph States and its Applications, Proceedings of the International School of Physics 
"Enrico Fermi" on "Quantum Computers, Algorithms and Chaos", Varenna, Italy (2005); quant-ph/0602096.

[30] M. Hein, W. Dür, and H.-J. Briegel, Phys. Rev. A 71, 032350 (2005).

[31] O. Gühne, B. Jungnitsch, T. Moroder, and Y.S. Weinstein, Phys. Rev. A 84, 052319 (2011).

[32] R. F. Werner, Phys. Rev. A 40, 4277 (1989).

[33] T. Eggeling and R. F. Werner, Phys. Rev. A 63, 042111 (2001).

[34] A. Cabello, J. Mod. Opt. 50, 10049 (2003).

[35] R. M. Wilcox, J. Math. Phys. 8, 962 (1967).

[36] W. H. Press, S. A. Teukolsky, W. T. Vetterling, B. P. Flannery, Numerical Recipes 3rd Edition: The Art of Scientific Computing, Cambridge University Press, New York (2007)

[37] S. Boyd and L. Vandenberghe, Convex Optimization, Cambridge University Press 2004.

[38] Y. S. Teo, H. Zhu, B.-G. Englert, J. Řeháček and Z. Hradil, Phys. Rev. Lett. 107, 020404 (2011). 